\title{
Output Feedback Adaptive Dynamic Surface Control of Permanent Magnet Synchronous Motor with Uncertain Time Delays via RBFNN
}

\author{
Shaohua Luo, ${ }^{1,2}$ Jiaxu Wang, ${ }^{1}$ Zhen Shi, ${ }^{1}$ and Qian Qiu ${ }^{1}$ \\ ${ }^{1}$ State Key Laboratory of Mechanical Transmission, Chongqing University, Chongqing 400044, China \\ ${ }^{2}$ Department of Mechanical Engineering, Chongqing Aerospace Polytechnic College, Chongqing 400021, China \\ Correspondence should be addressed to Shaohua Luo; hua66com@163.com
}

Received 25 July 2013; Revised 26 November 2013; Accepted 3 December 2013; Published 2 January 2014

Academic Editor: M. De la Sen

Copyright ( $\odot 2014$ Shaohua Luo et al. This is an open access article distributed under the Creative Commons Attribution License, which permits unrestricted use, distribution, and reproduction in any medium, provided the original work is properly cited.

\begin{abstract}
This paper focuses on an adaptive dynamic surface control based on the Radial Basis Function Neural Network for a fourth-order permanent magnet synchronous motor system wherein the unknown parameters, disturbances, chaos, and uncertain time delays are presented. Neural Network systems are used to approximate the nonlinearities and an adaptive law is employed to estimate accurate parameters. Then, a simple and effective controller has been obtained by introducing dynamic surface control technique on the basis of first-order filters. Asymptotically tracking stability in the sense of uniformly ultimate boundedness is achieved in a short time. Finally, the performance of the proposed control has been illustrated through simulation results.
\end{abstract}

\section{Introduction}

Recently, the permanent magnet synchronous motor (PMSM) is the most widely used driven mechanism because of the advantageous merits of cost, reliability, and performances. The PMSM is characterized by complexity, high nonlinearity, time-varying dynamics, inaccessibility of some states, and output for measurements; hence, it can be considered as a challenging engineering problem $[1,2]$. It is found that the PMSM is experiencing chaotic behavior at specific parameters and working conditions $[3,4]$. Then, the intermittent oscillation of torque and rotational speed, irregular current noise of the system, and unstable control performance appear in the PMSM, which seriously affect the stability and safety. Thus, it is difficult to accomplish the high-performance control of PMSM by using classic PID-type control methods.

A neuron-fuzzy controller (NFC) [5] is suitable for control of systems with uncertainties and nonlinearities. The NFC approach can also achieve self-learning; however, it is unsuitable for online learning real-time control due to the drawback of time consuming [6,7]. The sliding mode control (SMC) [8] can guarantee the robustness only under the bounds of the uncertainties and it has a shortage named chattering. The terminal sliding mode control (TSMC) method can assure convergence to the origin in finite time. Hence, the TSMC is successfully applied to PMSM driver system to improve control performance [9]. A position tracking control method via adaptive fuzzy backstepping is presented for the induction motors with unknown parameters [10]. Unfortunately, the traditional backstepping suffers from the "explosion of complexity" caused by the repeated differentiation of virtual control functions [11]. In order to overcome the above shortcomings, a backstepping approach combined with SMC technique is presented to realize synchronization of uncertain fractional-order strict-feedback chaotic system [12].

The dynamic surface control (DSC) developed by Swaroop et al. [13] is a control technique by introducing a firstorder filter at each recursive step of the backstepping design procedure, so the differentiation items on the virtual function can be avoided. By incorporating DSC into a neural network-based adaptive control design framework, Wang and Huang [14] proposed a backstepping-based control design for a class of nonlinear systems in strict-feedback form with arbitrary uncertainty. Zhang and Ge [15] further studied the control design for some special nonlinear systems 


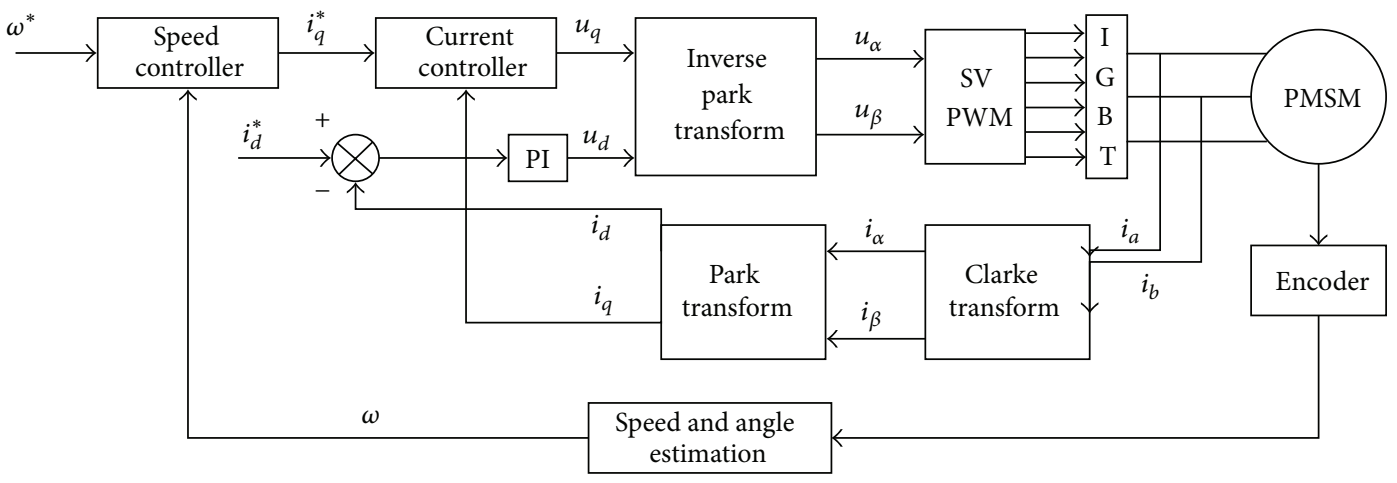

FIGURE 1: The principle block diagram of PMSM system based on vector control.

with unknown dead-zone using the DSC technique. To control the undesirable chaos in PMSM, an adaptive DSC controller was designed by introducing first-order low-pass filters [16], whereas in [17] DSC method was extended to time-delay uncertain nonlinear systems in parametric strictfeedback form. The new developments referring to DSC for different nonlinear systems and the applications to various engineering fields can be found in [18-20].

In this paper, motivated by the previous studies reported in the literature, the following problems will be addressed. An adaptive DSC based on the Radial Basis Function Neural Network (RBFNN) is discussed for the chaotic PMSM system. The whole process of the system design is performed in a step-by-step manner. For each step, a first-order filter is used to gain the information of derivative of the virtual control function and RBFNN is adopted to approximate the nonlinearity. The uncertain parameters in the system are updated by adaptive laws, which realize accurate parameters estimate. Then, an adaptive virtual control law is designed to stabilize each subsystem in the former steps. Finally, an adaptive DSC is employed via RBFNN in the last step to stabilize the whole system. Further contributions include the design of controller to handle uncertain time delays and disturbances.

\section{Dynamics of the PMSM System}

The model of a PMSM can be described in the well-known $(d-q)$ frame through the Park transformation as follows. The stator $d, q$ equations in the rotor frame are expressed as follows [21, 22]:

$$
\begin{gathered}
\frac{d \omega}{d t}=\frac{3}{2 J} n_{p}\left[\left(L_{d}-L_{q}\right) i_{d} i_{q}+\Phi i_{q}\right]-\frac{B}{J} \omega-\frac{T_{L}}{J}, \\
\frac{d i_{d}}{d t}=-\frac{R_{s}}{L_{d}} i_{d}+\frac{n_{p} L_{q}}{L_{d}} \omega i_{q}+\frac{1}{L_{d}} u_{d}, \\
\frac{d i_{q}}{d t}=-\frac{R_{s}}{L_{q}} i_{q}-\frac{n_{p} L_{d}}{L_{q}} \omega i_{d}-\frac{n_{p} \Phi}{L_{q}} \omega+\frac{1}{L_{q}} u_{q}, \\
\frac{d \theta}{d t}=\omega,
\end{gathered}
$$

where $u_{d}$ and $u_{q}$ are the $d-q$ axis voltages, which denote the system control input. $i_{d}, i_{q}, \theta$, and $\omega$ are state variables, which stand for the $d-q$ axis currents, the rotor position, and rotor angular velocity, respectively. $n_{p}, J$, and $B$ mean the pole pair, moment of inertia, and viscous friction coefficient. $L_{q}$ and $L_{r}$ denote the inductance of the stator in the $(d-q)$ frame. $T_{L}, R_{S}$, and $\Phi$ stand for the load torque, stator resistance, and magnet flux linkage of inertia.

The general schematic of the PMSM is depicted in Figure 1. The overall system consists of a PMSM with load, space vector pulse width modulation (SVPWM), fieldorientation mechanism, voltage-source inverter (VSI), and three controllers. The controllers employ a structure of cascade control loop including a speed loop and two current loops. PI controller, which is used to stabilize the $d$-axis current errors of the vector controlled drive, is adopted in the $d$-axis current loop. From Figure 1, the rotor angular velocity $\omega$ can be obtained from the position and speed sensor. The currents $i_{d}$ and $i_{q}$ can be calculated from $i_{a}$ and $i_{b}$ by Clarke and Park transformations. However, in real applications, this method suffers from serious problems, including internal disturbance such as torque ripples, parameters variation, friction forces, and unmodeled dynamics and external disturbance such as load disturbance. Thus, an adaptive dynamic surface controller via RBFNN is developed to solve these problems which can degrade the performance of closed-loop system if the controller does not have enough ability to reject them.

Equation (1) are rewritten as follows:

$$
\begin{gathered}
\frac{d \bar{\omega}}{d \bar{t}}=\sigma\left(\bar{i}_{q}-\bar{\omega}\right)-\bar{T}_{L}, \\
\frac{d \bar{i}_{q}}{d \bar{t}}=-\bar{i}_{q}-\bar{i}_{d} \bar{\omega}+\gamma \bar{\omega}+\bar{u}_{q}, \\
\frac{d \bar{i}_{d}}{d \bar{t}}=-\bar{i}_{d}+\bar{i}_{q} \bar{\omega}+\bar{u}_{d}, \\
\frac{d \bar{\theta}}{d \bar{t}}=\bar{\omega},
\end{gathered}
$$


where $\gamma=-\Phi /(\kappa L), \sigma=B \tau / J, \bar{T}_{L}=\tau^{2} T_{L} / J, \bar{u}_{q}=u_{q} /(\kappa R)$, $\bar{u}_{d}=u_{d} /(\kappa R), \tau=L / R, \kappa=B /\left(n_{p} \tau \Phi\right), \bar{\theta}=\theta, \bar{\omega}=\omega \tau, \bar{i}_{d}=$ $i_{d} / \kappa, \bar{i}_{q}=i_{q} / \kappa, \bar{t}=t / \tau, L=L_{d}=L_{q}$.

The chaotic behavior is demonstrated in PMSM system when its parameters fall into a certain area. Figures 2 and 3 show the strange attractor and the chaotic time series of the PMSM under the condition of $\sigma=5.46, \gamma=20, \bar{u}_{q}=\bar{u}_{d}=0$, $\bar{T}_{L}=0, \bar{\theta}(0)=-1, \bar{\omega}(0)=-5, \bar{i}_{q}(0)=0.01$, and $\bar{i}_{d}(0)=20$, in which the PMSM appears an aperiodic, random, sudden, or intermittent morbid oscillation.

For simplicity, the following notations are introduced:

$$
\begin{gathered}
x_{1}=\theta, \quad x_{2}=\omega, \quad x_{3}=i_{q}, \quad x_{4}=i_{d}, \\
a_{1}=\frac{3}{2} n_{p} \Phi, \quad a_{2}=\frac{3}{2} n_{p}\left(L_{d}-L_{q}\right), \\
b_{1}=-\frac{R_{s}}{L_{q}}, \quad b_{2}=-\frac{n_{p} L_{d}}{L_{q}}, \\
b_{3}=-\frac{n_{p} \Phi}{L_{q}}, \quad b_{4}=\frac{1}{L_{q}}, \\
c_{1}=-\frac{R_{s}}{L_{d}}, \quad c_{2}=\frac{n_{p} L_{q}}{L_{d}}, \quad c_{3}=\frac{1}{L_{d}} .
\end{gathered}
$$

By using these notations, the dynamic model of PMSM with uncertain time delays and disturbance can be described by the following differential equations:

$$
\begin{gathered}
\dot{x}_{1}=x_{2}+\Delta f_{1}\left(\bar{x}_{1}\left(t-\tau_{1}\right)\right)+d_{1}, \\
\dot{x}_{2}=\frac{a_{1}}{J} x_{3}+\frac{a_{2}}{J} x_{3} x_{4}-\frac{B}{J} x_{2}-\frac{T_{L}}{J}+\Delta f_{2}\left(\bar{x}_{2}\left(t-\tau_{2}\right)\right)+d_{2}, \\
\dot{x}_{3}=b_{1} x_{3}+b_{2} x_{2} x_{4}+b_{3} x_{2}+b_{4} u_{q}+\Delta f_{3}\left(\bar{x}_{3}\left(t-\tau_{3}\right)\right)+d_{3}, \\
\dot{x}_{4}=c_{1} x_{4}+c_{2} x_{2} x_{3}+c_{3} u_{d}+\Delta f_{4}\left(x_{4}\left(t-\tau_{4}\right)\right)+d_{4},
\end{gathered}
$$

where $\bar{x}_{i}(\cdot)=\left[x_{1}, \ldots, x_{i}\right]^{T}, i=1,2,3, \Delta f_{i}(\cdot), i=1, \ldots, 4$, denote uncertain nonlinear function owing to the effect of time delay, $\tau_{i}, i=1, \ldots, 4$, represent time-delay constants, and $d_{i}, i=1, \ldots, 4$, mean the disturbances.

Define the tracking errors

$$
\begin{gathered}
S_{1}(t)=x_{1}(t)-y_{r}, \\
S_{i}(t)=x_{i}(t)-\alpha_{i f}, \quad i=2,3 \\
S_{4}(t)=x_{4}(t),
\end{gathered}
$$

where $\alpha_{i f}, i=2,3$, will be given later by the first-order filter and $y_{r}$ is given continuous tracking signal.

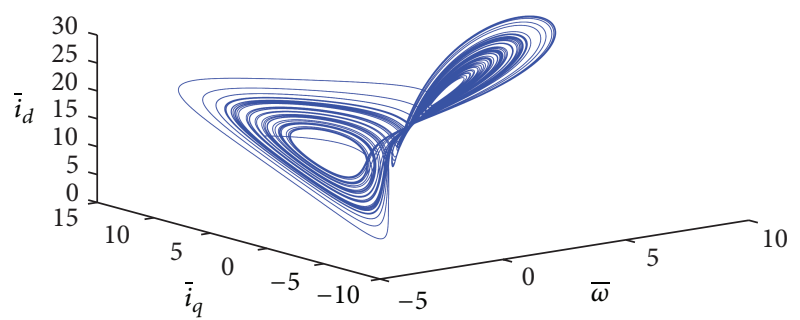

FIGURE 2: The strange attractor of the chaotic PMSM.

Then, for the uncertain nonlinear time-delay functions, the following inequalities can be satisfied:

$$
\begin{array}{r}
\left|\Delta f_{i}\left(\bar{x}_{i}\left(t-\tau_{i}\right)\right)\right| \leq \sum_{j=1}^{i}\left|S_{i}\left(t-\tau_{i}\right)\right| q_{i j}\left(\bar{S}_{i}\left(t-\tau_{i}\right)\right), \\
i=1, \ldots, 3, \\
\left|\Delta f_{4}\left(x_{4}\left(t-\tau_{4}\right)\right)\right| \leq\left|S_{4}\left(t-\tau_{4}\right)\right| q_{44}\left(S_{4}\left(t-\tau_{4}\right)\right),
\end{array}
$$

where $q_{i j}$ and $q_{44}$ are known continuous functions, $\bar{S}_{i}(\cdot)=$ $\left[S_{1}, \ldots, S_{i}\right]^{T}$.

In order to facilitate the design, the following assumption is given.

Assumption 1. $\left|g_{i}\right| \leq g_{M i}, i=1, \ldots, 3$, the desired trajectory $y_{r}$ is continuous, and its first-order derivative $y_{r}$ and secondorder derivative $\ddot{y}_{r}$ are bounded and available.

In this paper, the control objective is to force the tracking error $S_{1}(t)$ asymptotical stable in the sense of uniformly ultimate boundedness, which also ensures that the system state $x_{1}(t)$ can track a reference trajectory $y_{r}$.

\section{Adaptive Dynamic Surface Controller Based on RBFNN}

3.1. RBFNN System. The type of RBFNN shown in Figure 4 is considered as a two-layer network, which contains a hidden layer and an output layer. In this paper, the RBF neural network will be used to approximate the unknown continuous function $f(z): R^{n} \rightarrow R$ as

$$
\widehat{f}(z)=\theta^{* T} \xi(z),
$$

where $z \in \Omega \subset R^{n}$ is the input vector with $n$ being the neural network input dimension, $\theta^{*}=\left[\theta_{1}^{*}, \theta_{2}^{*}, \ldots, \theta_{l}^{*}\right]^{T} \in R^{l}$ is the weight vector, $l>1$ is the node number of neuron, and $\xi(z)=$ $\left[\xi_{1}(z), \xi_{2}(z), \ldots, \xi_{n}(z)\right]^{T} \in R^{l}$ is a basic function vector with $\xi_{i}(z)$ chosen as the commonly used Gaussian function in the following form:

$$
\xi_{i}(z)=\exp \left[-\frac{\left|z-\mu_{i}\right|^{2}}{2 \sigma_{i}^{2}}\right]=\exp \left[-\frac{\left(z-\mu_{i}\right)^{T}\left(z-\mu_{i}\right)}{2 \sigma_{i}^{2}}\right],
$$$$
i=1,2, \ldots, l \text {, }
$$ 


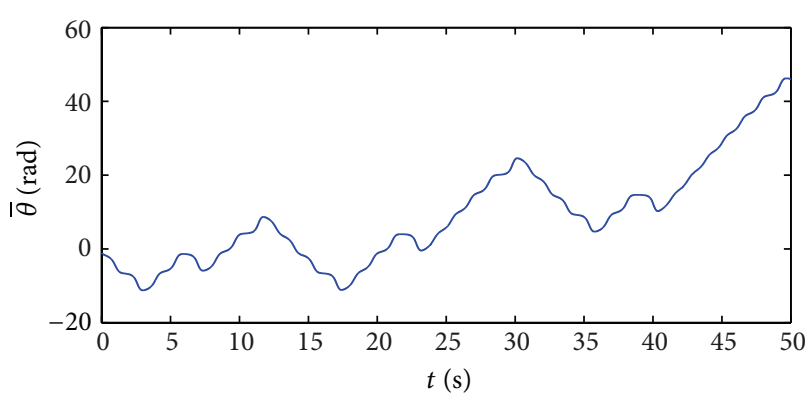

(a)

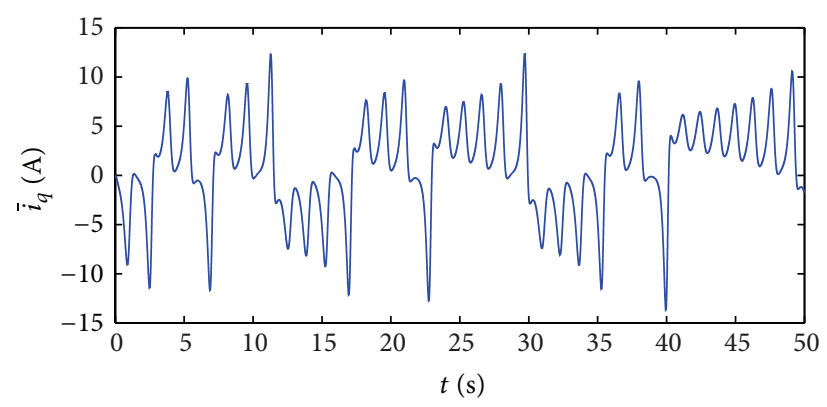

(c)

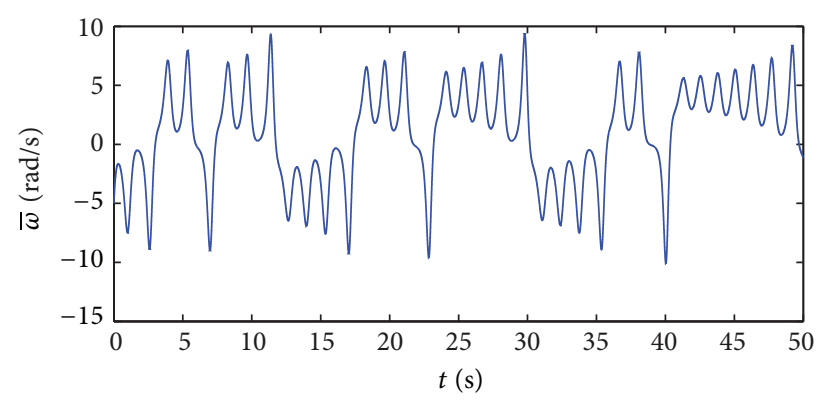

(b)

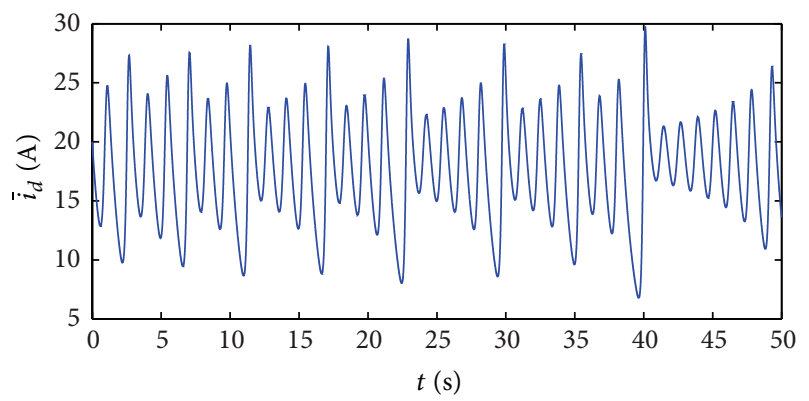

(d)

FIGURE 3: The chaotic time series of PMSM.

where $\mu_{i}=\left[\mu_{i 1}, \mu_{i 2}, \ldots, \mu_{i n}\right]^{T}$ is the center of the receptive field and $\sigma_{i}$ is the width of $\xi_{i}(z)$.

For given scalar $\varepsilon>0$, by choosing sufficiently large $l$, the RBFNN can approximate any continuous function over a compact set $\Omega \in R^{n}$ to arbitrary accuracy as

$$
f(z)=\theta^{T} \xi(z)+\delta(z), \quad \forall z \in \Omega \in R^{n},
$$

where $\delta(z)$ is the approximation error, satisfying $|\delta(z)|<\varepsilon$, and $\theta$ is an unknown ideal constant weight vector, which is an artificial quantity required for analytical purpose. Typically, $\theta$ is chosen as the value of $\theta^{*}$ that minimizes $|\delta(z)|$ for all $z \in \Omega$; that is,

$$
\theta=\arg \min _{\theta^{*} \in R^{n}}\left\{\sup _{z \in \Omega}\left|f(z)-\theta^{* T} \xi(z)\right|\right\} .
$$

3.2. Controller Design. In this section, an adaptive dynamic surface control approach of the permanent magnet synchronous motor based on RBF neural network will be developed. The DSC technique based on first-order filters is used to design the controller instead of the traditional backstepping method, and in sequence the explosion of terms problem is avoided.

In order to design dynamic surface controller, the boundary layer errors are defined as follows:

$$
y_{i}=\alpha_{i f}-\alpha_{i}, \quad i=2,3,
$$

where virtual control input $\alpha_{i}, i=2,3$, will be designed in the subsequent content.

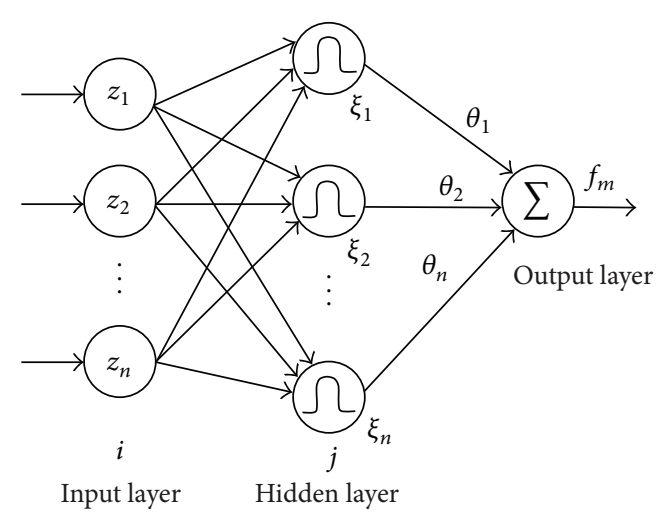

FIgURE 4: The structure of the RBFNN.

The designing process is as follows.

Step 1. Consider the tracking error $S_{1}=x_{1}-y_{r}$; the time derivative of $S_{1}$ is given by

$$
\dot{S}_{1}=x_{2}+\Delta f_{1}\left(\bar{x}_{1}\left(t-\tau_{1}\right)\right)+d_{1}-\dot{y}_{r} .
$$

Choose Lyapunov-Krasovskii functional as

$$
V_{1}=\frac{1}{2} S_{1}^{2}(t)+\sum_{j=1}^{3} \int_{t-\tau_{j}}^{t} S_{1}^{2}(\tau) q_{j 1}^{2}\left(\bar{S}_{1}(\tau)\right) d \tau
$$


The derivative of $V_{1}$ is

$$
\begin{aligned}
\dot{V}_{1}= & {\left[S_{2}+y_{2}+\alpha_{2}+d_{1}-\dot{y}_{r}+\Delta f_{1}\left(\bar{x}_{1}\left(t-\tau_{1}\right)\right)\right] S_{1} } \\
& +\sum_{j=1}^{3} S_{1}^{2}(t) q_{j 1}^{2}\left(\bar{S}_{1}(t)\right)-\sum_{j=1}^{3} S_{1}^{2}\left(t-\tau_{j}\right) q_{j 1}^{2}\left(\bar{S}_{1}\left(t-\tau_{j}\right)\right) .
\end{aligned}
$$

Using Young's inequality and inequality (6), the following inequalities can be obtained:

$$
\begin{gathered}
S_{1} y_{2} \leq \frac{1}{2} S_{1}^{2}+\frac{1}{2} y_{2}^{2}, \\
S_{1} \Delta f_{1}\left(\bar{x}_{1}\left(t-\tau_{1}\right)\right) \leq \frac{1}{4} S_{1}^{2}+S_{1}^{2}\left(t-\tau_{1}\right) q_{11}^{2}\left(\bar{S}_{1}\left(t-\tau_{1}\right)\right) .
\end{gathered}
$$

Thus, it follows immediately from substituting (15) into (14) that

$$
\begin{aligned}
\dot{V}_{1} \leq & {\left[S_{2}+\alpha_{2}+\frac{3}{4} S_{1}-\dot{y}_{r}+\sum_{j=1}^{3} S_{1}(t) q_{j 1}^{2}\left(\bar{S}_{1}(t)\right)\right] S_{1} } \\
& +\frac{1}{2} y_{2}^{2}+D_{1}
\end{aligned}
$$

where $D_{1}=S_{1}^{2}\left(t-\tau_{1}\right) q_{11}^{2}\left(\bar{S}_{1}\left(t-\tau_{1}\right)\right)-\sum_{j=1}^{3} S_{1}^{2}\left(t-\tau_{j}\right) q_{j 1}^{2}\left(\bar{S}_{1}(t-\right.$ $\left.\left.\tau_{j}\right)\right)$.

The virtual control function $\alpha_{2}$ is constructed as

$$
\alpha_{2}=-k_{1} S_{1}-\frac{3}{4} S_{1}+\dot{y}_{r}-\sum_{j=1}^{3} S_{1}(t) q_{j 1}^{2}\left(\bar{S}_{1}(t)\right),
$$

where design parameter $k_{1}>0$.

It is obtained that

$$
\dot{V}_{1} \leq-k_{1} S_{1}^{2}+S_{2} S_{1}+\frac{1}{2} y_{2}^{2}+D_{1} .
$$

Step 2. Let $\alpha_{2}$ be passed through a first-order filter as follows:

$$
\tau_{2} \dot{\alpha}_{2 f}+\alpha_{2 f}=\alpha_{2}, \quad \alpha_{2 f}(0)=\alpha_{2}(0),
$$

where $\tau_{2}$ is a time constant.

With (5) and (19), one has

$$
\dot{\alpha}_{2 f}=-\frac{y_{2}}{\tau_{2}} .
$$

The derivative of $y_{2}$ is

$$
\begin{aligned}
\dot{y}_{2}= & \dot{\alpha}_{2 f}-\dot{\alpha}_{2}=-\frac{y_{2}}{\tau_{2}} \\
& -\left(-k_{1} \dot{S}_{1}-\frac{3}{4} \dot{S}_{1}+\ddot{y}_{r}-\sum_{j=1}^{3} \dot{S}_{1}(t) q_{j 1}^{2}\left(\bar{S}_{1}(t)\right)\right. \\
& \left.-\sum_{j=1}^{3} S_{1}(t) \dot{q}_{j 1}^{2}\left(\bar{S}_{1}(t)\right)\right) .
\end{aligned}
$$

It is obtained that

$$
\left|\dot{y}_{2}+\frac{y_{2}}{\tau_{2}}\right| \leq B_{2}\left(S_{1}, S_{2}, y_{2}, q_{j 1}, y_{r}, \dot{y}_{r}, \ddot{y}_{r}\right), \quad j=1, \ldots, 3 \text {, }
$$

where $B_{2}\left(S_{1}, S_{2}, y_{2}, q_{j 1}, y_{r}, \dot{y}_{r}, \ddot{y}_{r}\right), j=1, \ldots, 3$, is the continuous function.

Using Young's inequality, one has

$$
y_{2} \dot{y}_{2} \leq-\frac{y_{2}^{2}}{\tau_{2}}+y_{2}^{2}+\frac{1}{4} B_{2}^{2}
$$

Differentiating $S_{2}$ gives

$$
\begin{aligned}
\dot{S}_{2}= & \frac{a_{1}}{J} x_{3}+\frac{a_{2}}{J} x_{3} x_{4}-\frac{B}{J} x_{2}-\frac{T_{L}}{J} \\
& +\Delta f_{2}\left(\bar{x}_{2}\left(t-\tau_{2}\right)\right)+d_{2}-\dot{\alpha}_{2 f} \\
= & g_{1} x_{3}+f_{1}+\Delta f_{2}\left(\bar{x}_{2}\left(t-\tau_{2}\right)\right)+d_{2}-\dot{\alpha}_{2 f},
\end{aligned}
$$

where $f_{1}=\left(a_{2} / J\right) x_{3} x_{4}-(B / J) x_{2}-T_{L} / J, g_{1}=a_{1} / J$.

In the realistic model of PMSM, limited to the working conditions, the parameter $g_{1}$ is unknown. So it cannot be used to construct the control signal. Thus, let $\widehat{g}_{1}$ be the estimation of $g_{1}$. Meanwhile, notice that the nonlinear function $f_{1}$ contains uncertain parameters, such as $a_{2}, B, J$, and $T_{L}$. This will make the traditional adaptive DSC design very troublesome. To avoid this trouble and simplify the control structure, the RBFNN will be employed to approximate $f_{1}$.

According to the description above, for any given $\varepsilon_{1}>0$, there exists a RBFNN system $\theta_{1}^{T} \xi_{1}$ such that

$$
f_{1}=\theta_{1}^{T} \xi_{1}+\delta_{1}
$$

where $\delta_{1}$ is the approximation error and satisfies $\left|\delta_{1}\right|<\varepsilon_{1}$.

With (24) and (25), one has

$$
\dot{S}_{2}=g_{1} x_{3}+\theta_{1}^{T} \xi_{1}+\delta_{1}+\Delta f_{2}\left(\bar{x}_{2}\left(t-\tau_{2}\right)\right)+d_{2}-\dot{\alpha}_{2 f} \text {. }
$$

Consider the Lyapunov-Krasovskii functional candidate $V_{2}$ as

$$
\begin{aligned}
V_{2}= & V_{1}+\frac{1}{2} S_{2}^{2}+\frac{1}{2} y_{2}^{2}+\frac{1}{2 \gamma_{1}}\left(\widehat{\theta}_{1}-\theta_{1}\right)^{T}\left(\widehat{\theta}_{1}-\theta_{1}\right) \\
& +\frac{1}{2 \Gamma_{1}}\left(\widehat{g}_{1}-g_{1}\right)^{2}+\sum_{j=2}^{3} \int_{t-\tau_{j}}^{t} S_{2}^{2}(\tau) q_{j 2}^{2}\left(\bar{S}_{2}(\tau)\right) d \tau .
\end{aligned}
$$

Then, the derivative of $V_{2}$ is given by

$$
\begin{gathered}
\dot{V}_{2} \leq \dot{V}_{1}+S_{2}\left[g_{1}\left(S_{3}+y_{3}+\alpha_{3}\right)+\theta_{1}^{T} \xi_{1}+\delta_{1}+d_{2}-\dot{\alpha}_{2 f}\right] \\
+S_{2} \Delta f_{2}\left(\bar{x}_{2}\left(t-\tau_{2}\right)\right)+\left(1-\frac{1}{\tau_{2}}\right) y_{2}^{2}+\frac{1}{4} B_{2}^{2}+\frac{1}{\gamma_{1}} \\
\quad \times\left(\widehat{\theta}_{1}-\theta_{1}\right)^{T} \dot{\hat{\theta}}_{1}+\frac{1}{\Gamma_{1}}\left(\widehat{g}_{1}-g_{1}\right) \dot{\hat{g}}_{1} \\
+\sum_{j=2}^{3} S_{2}^{2}(t) q_{j 2}^{2}\left(\bar{S}_{2}(t)\right)-\sum_{j=2}^{3} S_{2}^{2}\left(t-\tau_{j}\right) q_{j 2}^{2} \\
\times\left(\bar{S}_{2}\left(t-\tau_{j}\right)\right) .
\end{gathered}
$$


Using Young's inequality and inequality Assumption 1, the following inequalities can be obtained:

$$
\begin{gathered}
S_{2} y_{3} \leq \frac{1}{2} S_{2}^{2}+\frac{1}{2} y_{3}^{2} \\
S_{2} \Delta f_{2}\left(\bar{x}_{2}\left(t-\tau_{2}\right)\right) \leq \frac{1}{4} S_{2}^{2}+\sum_{j=1}^{2} S_{j}^{2}\left(t-\tau_{2}\right) q_{2 j}^{2}\left(\bar{S}_{j}\left(t-\tau_{2}\right)\right) .
\end{gathered}
$$

Substituting (29) into (28) gives

$$
\begin{aligned}
\dot{V}_{2} \leq S_{2}\left[g_{1}\left(S_{3}+\alpha_{3}\right)+\frac{1}{2} S_{2} g_{1}+S_{1}+\theta_{1}^{T} \xi_{1}+\delta_{1}\right. \\
\left.+d_{2}-\dot{\alpha}_{2 f}+\frac{1}{4} S_{2}+\sum_{j=2}^{3} S_{2}(t) q_{j 2}^{2}\left(\bar{S}_{2}(t)\right)\right] \\
-k_{1} S_{1}^{2}+\frac{1}{2} y_{2}^{2}+\frac{1}{2} g_{1} y_{3}^{2}+\left(1-\frac{1}{\tau_{2}}\right) y_{2}^{2}+\frac{1}{4} B_{2}^{2} \\
+\frac{1}{\gamma_{1}}\left(\widehat{\theta}_{1}-\theta_{1}\right)^{T} \dot{\hat{\theta}}_{1}+\frac{1}{\Gamma_{1}}\left(\widehat{g}_{1}-g_{1}\right) \dot{\hat{g}}_{1}+\sum_{j=1}^{2} D_{j},
\end{aligned}
$$

where $D_{2}=\sum_{j=1}^{2} S_{j}^{2}\left(t-\tau_{2}\right) q_{2 j}^{2}\left(\bar{S}_{j}\left(t-\tau_{2}\right)\right)-\sum_{j=2}^{3} S_{2}^{2}\left(t-\tau_{j}\right) q_{j 2}^{2}$ $\left(\bar{S}_{2}\left(t-\tau_{j}\right)\right)$.

Choose the virtual control function $\alpha_{3}$ and the corresponding parameters adaptive laws as

$$
\begin{gathered}
\alpha_{3}=\frac{\widehat{g}_{1}}{\widehat{g}_{1}^{2}+\eta_{1}}\left[-\left(k_{2}+\frac{1}{2} \widehat{g}_{1}+\frac{1}{4}\right) S_{2}-S_{1}-\widehat{\theta}_{1}^{T} \xi_{1}+\dot{\alpha}_{2 f}\right. \\
\left.\quad-\sum_{j=2}^{3} S_{2}(t) q_{j 2}^{2}\left(\bar{S}_{2}(t)\right)\right] \\
\dot{\hat{\theta}}_{1}=\gamma_{1}\left(\xi_{1} S_{2}-m_{1} \widehat{\theta}_{1}\right) \\
\dot{\hat{g}}_{1}=\Gamma_{1}\left(S_{2} \alpha_{3}-c_{1} \widehat{g}_{1}\right)
\end{gathered}
$$

where $k_{2}>0, \gamma_{1}>0, m_{1}>0, \Gamma_{1}>0, c_{1}>0$, and $\eta_{1}>0$ are design parameters.

Substituting (31)-(33) into (30), (30) becomes

$$
\begin{array}{r}
\dot{V}_{2} \leq-\frac{\eta_{1}}{\widehat{g}_{1}^{2}+\eta_{1}}\left[-\left(k_{2}+\frac{1}{2} \widehat{g}_{1}+\frac{1}{4}\right) S_{2}-S_{1}-\widehat{\theta}_{1}^{T} \xi_{1}+\dot{\alpha}_{2 f}\right. \\
\left.-\sum_{j=2}^{3} S_{2}(t) q_{j 2}^{2}\left(\bar{S}_{2}(t)\right)\right] S_{2}\left(\delta_{1}+d_{2}\right) S_{2}
\end{array}
$$

$$
\begin{aligned}
& +g_{1} S_{2} S_{3}-\widetilde{\theta}_{1}^{T} \xi_{1} S_{2}-\widetilde{g}_{1} S_{2} \alpha_{3}-\sum_{j=1}^{2} k_{j} S_{j}^{2}-\frac{1}{2} S_{2}^{2} \widetilde{g}_{1}+\frac{1}{2} y_{2}^{2} \\
& +\frac{1}{2} g_{1} y_{3}^{2}+\left(1-\frac{1}{\tau_{2}}\right) y_{2}^{2}+\frac{1}{4} B_{2}^{2}+\left(\widehat{\theta}_{1}-\theta_{1}\right)^{T} \\
& \times\left(\xi_{1} S_{2}-m_{1} \widehat{\theta}_{1}\right)+\left(\widehat{g}_{1}-g_{1}\right)\left(S_{2} \alpha_{3}-c_{1} \widehat{g}_{1}\right)+\sum_{j=1}^{2} D_{j} \\
& \leq \frac{1}{4} \vartheta_{1}^{2}+S_{2}^{2}+g_{1} S_{2} S_{3}-\sum_{j=1}^{2} k_{j} S_{j}^{2}-\frac{1}{2} S_{2}^{2} \widetilde{g}_{1}+\frac{1}{2} y_{2}^{2}+\frac{1}{2} g_{1} y_{3}^{2} \\
& +\left(1-\frac{1}{\tau_{2}}\right) y_{2}^{2}+\frac{1}{4} B_{2}^{2}-m_{1} \widetilde{\theta}_{1}^{T} \widehat{\theta}_{1}-c_{1} \widetilde{g} \widehat{g}_{1}+\sum_{j=1}^{2} D_{j},
\end{aligned}
$$

where $\vartheta_{1} \geq \mid \varepsilon_{1}+d_{2}-\left(\eta_{1} /\left(\widehat{g}_{1}^{2}+\eta_{1}\right)\right)\left[-\left(k_{2}+(1 / 2) \widehat{g}_{1}+1 / 4\right) S_{2}-S_{1}-\right.$ $\left.\widehat{\theta}_{1}^{T} \xi_{1}+\dot{\alpha}_{2 f}-\sum_{j=2}^{3} S_{2}(t) q_{j 2}^{2}\left(\bar{S}_{2}(t)\right)\right] \mid$ is a continuous function.

Step 3. Filter $\alpha_{3}$ through the following first-order filter

$$
\tau_{3} \dot{\alpha}_{3 f}+\alpha_{3 f}=\alpha_{3}, \quad \alpha_{3 f}(0)=\alpha_{3}(0),
$$

where $\tau_{3}$ is a time constant.

With (5) and (35), one has

$$
\dot{\alpha}_{3 f}=-\frac{y_{3}}{\tau_{3}} .
$$

The derivative of $y_{3}$ is

$$
\begin{aligned}
& \dot{y}_{3}=\dot{\alpha}_{3 f}-\dot{\alpha}_{3} \\
& =-\frac{y_{3}}{\tau_{3}}-\left\{\frac { \widehat { g } _ { 1 } } { \widehat { g } _ { 1 } ^ { 2 } + \eta _ { 1 } } \left[-\left(k_{2}+\frac{1}{2} \widehat{g}_{1}+\frac{1}{4}\right) \dot{S}_{2}-\dot{S}_{1}\right.\right. \\
& -\dot{\hat{\theta}}_{1}^{T} \xi_{1}-\widehat{\theta}_{1}^{T} \frac{\partial \xi_{1}}{\partial x_{3}} \dot{x}_{3}+\ddot{\alpha}_{2 f} \\
& -\sum_{j=2}^{3} \dot{S}_{2}(t) q_{j 2}^{2}\left(\bar{S}_{2}(t)\right) \\
& \left.-\sum_{j=2}^{3} S_{2}(t) \dot{q}_{j 2}^{2}\left(\bar{S}_{2}(t)\right)\right] \\
& -\frac{\left(\eta_{1}-\widehat{g}_{1}^{2}\right) \dot{\hat{g}}_{1}}{\left(\hat{g}_{1}^{2}+\eta_{1}\right)^{2}} \\
& \times\left[-\left(k_{2}+\frac{1}{2} \widehat{g}_{1}+\frac{1}{4}\right) S_{2}-S_{1}-\widehat{\theta}_{1}^{T} \xi_{1}+\dot{\alpha}_{2 f}\right. \\
& \left.\left.-\sum_{j=2}^{3} S_{2}(t) q_{j 2}^{2}\left(\bar{S}_{2}(t)\right)\right]\right\}
\end{aligned}
$$


It is obtained that

$$
\begin{gathered}
\left|\dot{y}_{3}+\frac{y_{3}}{\tau_{3}}\right| \leq B_{3}\left(S_{1}, \ldots, s_{3}, y_{2}, y_{2}, q_{11}, \ldots, q_{31},\right. \\
\left.q_{22}, q_{32}, \widehat{\theta}_{1}, \widehat{g}_{1}, y_{r}, \dot{y}_{r}, \ddot{y}_{r}\right),
\end{gathered}
$$

where $B_{3}\left(S_{1}, \ldots, S_{3}, y_{2}, y_{2}, q_{11}, \ldots, q_{31}, q_{22}, q_{32}, \widehat{\theta}_{1}, \widehat{g}_{1}, y_{r}, \dot{y}_{r}\right.$, $\ddot{y}_{r}$ ) is the continuous function.

Using Young's inequality, one has

$$
y_{3} \dot{y}_{3} \leq-\frac{y_{3}^{2}}{\tau_{3}}+y_{3}^{2}+\frac{1}{4} B_{3}^{2} \text {. }
$$

Differentiating $S_{3}$ results in the following equation:

$$
\dot{S}_{3}=f_{2}+b_{4} u_{q}+\Delta f_{3}\left(\bar{x}_{3}\left(t-\tau_{3}\right)\right)+d_{3}-\dot{\alpha}_{3 f},
$$

where $f_{2}=b_{1} x_{3}+b_{2} x_{2} x_{4}+b_{3} x_{2}$.

Notice that the unknown parameters, such as $b_{1}, b_{2}$, and $b_{3}$, appear in the expression of $f_{2}$. To facilitate the design of controller, the RBFNN will be employed to approximate the nonlinear function $f_{2}$.

According to (9), for any given $\varepsilon_{2}>0$, there exists a neural network $\theta_{2}^{T} \xi_{2}$ such that

$$
f_{2}=\theta_{2}^{T} \xi_{2}+\delta_{2}
$$

where $\delta_{2}$ is the approximation error and satisfies $\left|\delta_{2}\right| \leq \varepsilon_{2}$.

Thus, (40) is rewritten as follows:

$$
\dot{S}_{3}=\theta_{2}^{T} \xi_{2}+\delta_{2}+b_{4} u_{q}+\Delta f_{3}\left(\bar{x}_{3}\left(t-\tau_{3}\right)\right)+d_{3}-\dot{\alpha}_{3 f}
$$

Define Lyapunov-Krasovskii functional

$$
\begin{aligned}
V_{3}= & V_{2}+\frac{1}{2} S_{3}^{2}+\frac{1}{2} y_{3}^{2}+\frac{1}{2 \gamma_{2}}\left(\widehat{\theta}_{2}-\theta_{2}\right)^{T}\left(\widehat{\theta}_{2}-\theta_{2}\right) \\
& +\frac{1}{2 \Gamma_{2}}\left(\widehat{g}_{2}-g_{2}\right)^{2}+\int_{t-\tau_{3}}^{t} S_{3}^{2}(\tau) q_{33}^{2}\left(\bar{S}_{3}(\tau)\right) d \tau .
\end{aligned}
$$

Then, the derivative of $V_{3}$ is computed by

$$
\begin{aligned}
\dot{V}_{3} \leq & \dot{V}_{2}+S_{3}\left[g_{2} u_{q}+\theta_{2}^{T} \xi_{2}+\delta_{2}+d_{2}-\dot{\alpha}_{3 f}\right] \\
& +S_{3} \Delta f_{3}\left(\bar{x}_{3}\left(t-\tau_{3}\right)\right)+\left(1-\frac{1}{\tau_{3}}\right) y_{3}^{2}+\frac{1}{4} B_{3}^{2} \\
& +\frac{1}{\gamma_{2}}\left(\widehat{\theta}_{2}-\theta_{2}\right)^{T} \dot{\hat{\theta}}_{2}+\frac{1}{\Gamma_{2}}\left(\widehat{g}_{2}-g_{2}\right) \dot{\hat{g}}_{2} \\
& +S_{3}^{2}(t) q_{33}^{2}\left(\bar{S}_{3}(t)\right)-S_{3}^{2}\left(t-\tau_{3}\right) q_{33}^{2}\left(\bar{S}_{3}\left(t-\tau_{3}\right)\right),
\end{aligned}
$$

where $g_{2}=b_{4}$.

Using Young's inequality and inequality (6), one has

$$
S_{3} \Delta f_{3}\left(\bar{x}_{3}\left(t-\tau_{3}\right)\right) \leq \frac{1}{4} S_{3}^{2}+\sum_{j=1}^{3} S_{j}^{2}\left(t-\tau_{3}\right) q_{3 j}^{2}\left(\bar{S}_{j}\left(t-\tau_{3}\right)\right) .
$$

Thus, it follows immediately from substituting (45) into (44) that

$$
\begin{aligned}
\dot{V}_{3} \leq & S_{3}\left[g_{2} u_{q}+\theta_{2}^{T} \xi_{2}+\delta_{2}+d_{2}-\dot{\alpha}_{3 f}+\frac{1}{4} S_{3}+S_{3}(t) q_{33}^{2}\right. \\
& \left.\times\left(\bar{S}_{3}(t)\right)+g_{1} S_{2}\right]+\frac{1}{4} \vartheta_{1}^{2}+\frac{1}{\gamma_{2}}\left(\widehat{\theta}_{2}-\theta_{2}\right)^{T} \dot{\hat{\theta}}_{2} \\
+ & \frac{1}{\Gamma_{2}}\left(\widehat{g}_{2}-g_{2}\right) \dot{\hat{g}}_{2}+S_{2}^{2}-\frac{1}{2} S_{2}^{2} \widetilde{g}_{1}+\frac{1}{2} y_{2}^{2}+\frac{1}{2} g_{1} y_{3}^{2} \\
& -m_{1} \widetilde{\theta}_{1}^{T} \widehat{\theta}_{1}-c_{1} \widetilde{g}_{j} \widehat{g}_{j}-\sum_{j=1}^{2} k_{j} S_{j}^{2}+\sum_{j=2}^{3}\left(1-\frac{1}{\tau_{j}}\right) y_{j}^{2} \\
+ & \sum_{j=2}^{3} \frac{1}{4} B_{j}^{2}+\sum_{j=1}^{3} D_{j},
\end{aligned}
$$

where $D_{3}=\sum_{j=1}^{3} S_{j}^{2}\left(t-\tau_{3}\right) q_{3 j}^{2}\left(\bar{S}_{j}\left(t-\tau_{3}\right)\right)-S_{3}^{2}\left(t-\tau_{3}\right) q_{33}^{2}\left(\bar{S}_{3}(t-\right.$ $\left.\left.\tau_{3}\right)\right)$.

At the present stage, the control law $u_{q}$ and the related parameters adaptive laws are designed as

$$
\begin{aligned}
u_{q}=\frac{\widehat{g}_{2}}{\widehat{g}_{2}^{2}+\eta_{2}}[ & -\left(k_{3}+\frac{1}{4}\right) S_{3}-\widehat{\theta}_{2}^{T} \xi_{2}-g_{1} S_{2} \\
& \left.+\dot{\alpha}_{3 f}-S_{3}(t) q_{33}^{2}\left(\bar{S}_{3}(t)\right)\right], \\
\dot{\hat{\theta}}_{2}= & \gamma_{2}\left(\xi_{2} S_{3}-m_{2} \widehat{\theta}_{2}\right), \\
\dot{\hat{g}}_{2}= & \Gamma_{2}\left(S_{3} u_{q}-c_{2} \widehat{g}_{2}\right),
\end{aligned}
$$

where $k_{3}>0, \gamma_{2}>0, m_{2}>0, \Gamma_{2}>0, c_{2}>0$, and $\eta_{2}>0$ are design parameters. 

has

By similar manipulations previously done in Step 2, one

$$
\begin{aligned}
\dot{V}_{3} \leq & \left(\delta_{2}+d_{3}\right) S_{3}-\frac{\eta_{2}}{\widehat{g}_{2}^{2}+\eta_{2}}\left[-\left(k_{3}+\frac{1}{4}\right) S_{3}-\widehat{\theta}_{2}^{T} \xi_{2}-g_{1} S_{2}\right. \\
& \left.+\dot{\alpha}_{3 f}-S_{3}(t) q_{33}^{2}\left(\bar{S}_{3}(t)\right)\right] S_{3} \\
+ & \frac{1}{4} \vartheta_{1}^{2}-k_{3} S_{3}^{2}-\widetilde{\theta}_{2}^{T} \xi_{2} S_{3}-\widetilde{g}_{2} u_{q} S_{3}+\widetilde{\theta}_{2}^{T}\left(\xi_{2} S_{3}-m_{2} \widehat{\theta}_{2}\right) \\
+ & \widetilde{g}_{2}\left(S_{3} u_{q}-c_{2} \widehat{g}_{2}\right)+S_{2}^{2}-\frac{1}{2} S_{2}^{2} \widetilde{g}_{1}+\frac{1}{2} y_{2}^{2}+\frac{1}{2} g_{1} y_{3}^{2} \\
& -m_{1} \widetilde{\theta}_{1}^{T} \widehat{\theta}_{1}-c_{1} \widehat{g}_{1} \widetilde{g}_{1}-\sum_{j=1}^{2} k_{j} S_{j}^{2}+\sum_{j=2}^{3}\left(1-\frac{1}{\tau_{j}}\right) y_{j}^{2} \\
& +\sum_{j=2}^{3} \frac{1}{4} B_{j}^{2}+\sum_{j=1}^{3} D_{j} \leq \sum_{j=1}^{2} \frac{1}{4} \vartheta_{j}^{2}+\sum_{j=1}^{2} S_{j}^{2}-\frac{1}{2} S_{2}^{2} \widetilde{g}_{1}+\frac{1}{2} y_{2}^{2} \\
& +\frac{1}{2} g_{1} y_{3}^{2}-\sum_{j=1}^{2} m_{j} \widetilde{\theta}_{j}^{T} \widehat{\theta}_{j}-\sum_{j=1}^{2} c_{j} \widetilde{g}_{j} \widehat{g}_{j}-\sum_{j=1}^{3} k_{j} S_{j}^{2} \\
& +\sum_{j=2}^{3}\left(1-\frac{1}{\tau_{j}}\right) y_{j}^{2}+\sum_{j=2}^{3} \frac{1}{4} B_{j}^{2}+\sum_{j=1}^{3} D_{j},
\end{aligned}
$$

where $\vartheta_{2} \geq \mid \varepsilon_{2}+d_{3}-\left(\eta_{2} /\left(\hat{g}_{2}^{2}+\eta_{2}\right)\right)\left[-\left(k_{3}+1 / 4\right) S_{3}-\widehat{\theta}_{2}^{T} \xi_{2}-\right.$ $\left.g_{1} S_{2}+\dot{\alpha}_{3 f}-S_{3}(t) q_{33}^{2}\left(\bar{S}_{3}(t)\right)\right] \mid$ is a continuous function.

Step 4. At this step, the control law $u_{d}$ will be constructed. In this end, choose Lyapunov-Krasovskii functional as $V_{4}=V_{3}$ $+(1 / 2) S_{4}^{2}+\left(1 / 2 \gamma_{3}\right)\left(\widehat{\theta}_{3}-\theta_{3}\right)^{T}\left(\widehat{\theta}_{3}-\theta_{3}\right)+\left(1 / 2 \Gamma_{3}\right)\left(\widehat{g}_{3}-g_{3}\right)^{2}+$ $\int_{t-\tau_{4}}^{t} S_{4}^{2}(\tau) q_{44}^{2}\left(S_{4}(\tau)\right) d \tau$. Then, the derivative of $V_{4}$ is given by

$$
\begin{aligned}
\dot{V}_{4}= & \dot{V}_{3}+S_{4}\left[f_{3}+g_{3} u_{d}+d_{4}+\Delta f_{4}\left(x_{4}\left(t-\tau_{4}\right)\right)\right] \\
& +\frac{1}{\gamma_{3}}\left(\widehat{\theta}_{3}-\theta_{3}\right)^{T} \dot{\tilde{\theta}}_{3}+\frac{1}{\Gamma_{3}}\left(\widehat{g}_{3}-g_{3}\right) \dot{\tilde{g}}_{3}+S_{4}^{2}(t) q_{44}^{2} \\
& \times\left(S_{4}(t)\right)-S_{4}^{2}\left(t-\tau_{4}\right) q_{44}^{2}\left(S_{4}\left(t-\tau_{4}\right)\right),
\end{aligned}
$$

where $f_{3}=c_{1} x_{4}+c_{2} x_{2} x_{3}, g_{3}=c_{3}$.

Similarly, the RBFNN system $\theta_{3}^{T} \xi_{3}$ is utilized to approximate the nonlinear function $f_{3}$ with unknown parameters $c_{1}$ and $c_{2}$. For any given $\varepsilon_{3}>0$, one has

$$
f_{3}=\theta_{3}^{T} \xi_{3}+\delta_{3}
$$

where $\delta_{3}$ is the approximation error and satisfies $\left|\delta_{3}\right|<\varepsilon_{3}$.

Note the fact that

$$
S_{4} \Delta f_{4}\left(x_{4}\left(t-\tau_{4}\right)\right) \leq \frac{1}{4} S_{4}^{2}+S_{4}^{2}\left(t-\tau_{4}\right) q_{44}^{2}\left(S_{4}\left(t-\tau_{4}\right)\right) .
$$

Substituting (52)-(53) into (51) gives

$$
\begin{aligned}
\dot{V}_{4} \leq & S_{4}\left[g_{3} u_{d}+\theta_{3}^{T} \xi_{3}+\delta_{3}+d_{4}+\frac{1}{4} S_{4}+S_{4}(t) q_{44}^{2}\left(S_{4}(t)\right)\right] \\
& +\frac{1}{\gamma_{3}}\left(\widehat{\theta}_{3}-\theta_{3}\right)^{T} \dot{\tilde{\theta}}_{3}+\frac{1}{\Gamma_{3}}\left(\widehat{g}_{3}-g_{3}\right) \dot{\tilde{g}}_{3}+\sum_{j=1}^{2} \frac{1}{4} \vartheta_{j}^{2}+\sum_{j=1}^{2} S_{j}^{2} \\
& -\frac{1}{2} S_{2}^{2} \widetilde{g}_{1}+\frac{1}{2} y_{2}^{2}+\frac{1}{2} g_{1} y_{3}^{2}-\sum_{j=1}^{2} m_{j} \widetilde{\theta}_{j}^{T} \widehat{\theta}_{j}-\sum_{j=1}^{2} c_{j} \widetilde{g}_{j} \widehat{g}_{j} \\
& -\sum_{j=1}^{3} k_{j} S_{j}^{2}+\sum_{j=2}^{3}\left(1-\frac{1}{\tau_{j}}\right) y_{j}^{2}+\sum_{j=2}^{3} \frac{1}{4} B_{j}^{2}+\sum_{j=1}^{4} D_{j},
\end{aligned}
$$

where $D_{4}=S_{4}^{2}\left(t-\tau_{4}\right) q_{44}^{2}\left(S_{4}\left(t-\tau_{4}\right)\right)-S_{4}^{2}\left(t-\tau_{4}\right) q_{44}^{2}\left(S_{4}\left(t-\tau_{4}\right)\right)$.

Now design $u_{d}, \dot{\hat{\theta}}_{3}$, and $\dot{\hat{g}}_{3}$ as

$$
u_{d}=\frac{\widehat{g}_{3}}{\widehat{g}_{3}^{2}+\eta_{3}}\left[-\left(k_{4}+\frac{1}{4}\right) S_{4}-\widehat{\theta}_{3}^{T} \xi_{3}-S_{4}(t) q_{44}^{2}\left(S_{4}(t)\right)\right],
$$

$$
\begin{aligned}
& \dot{\hat{\theta}}_{3}=\gamma_{3}\left(\xi_{3} S_{4}-m_{3} \widehat{\theta}_{3}\right), \\
& \dot{\hat{g}}_{3}=\Gamma_{3}\left(S_{4} u_{d}-c_{3} \widehat{g}_{3}\right),
\end{aligned}
$$

where $k_{4}>0, \gamma_{3}>0, m_{3}>0, \Gamma_{3}>0, c_{3}>0$, and $\eta_{3}>0$ are design parameters.

By similar manipulations previously done in Step 2, one has

$$
\begin{aligned}
\dot{V}_{4} \leq & \sum_{j=1}^{3} \frac{1}{4} \vartheta_{j}^{2}+\sum_{j=1}^{2} S_{j}^{2}-\frac{1}{2} S_{2}^{2} \widetilde{g}_{1}+\frac{1}{2} y_{2}^{2}+\frac{1}{2} g_{1} y_{3}^{2} \\
& -\sum_{j=1}^{4} k_{j} S_{j}^{2}-\sum_{j=1}^{3} m_{j} \widetilde{\theta}_{j}^{T} \widehat{\theta}_{j}-\sum_{j=1}^{3} c_{j} \widetilde{g}_{j} \widehat{g}_{j} \\
& +\sum_{j=2}^{3}\left(1-\frac{1}{\tau_{j}}\right) y_{j}^{2}+\sum_{j=2}^{3} \frac{1}{4} B_{j}^{2}+\sum_{j=1}^{4} D_{j},
\end{aligned}
$$

where $\vartheta_{3} \geq \mid \delta_{3}+d_{4}-\left(\eta_{3} /\left(\hat{g}_{3}^{2}+\eta_{3}\right)\right)\left[-\left(k_{4}+1 / 4\right) S_{4}-\widehat{\theta}_{3}^{T} \xi_{3}-\right.$ $\left.S_{4}(t) q_{44}^{2}\left(S_{4}(t)\right)\right] \mid$ is a continuous function.

Up to now, the whole design process of the controller of PMSM is completed, and the configuration of the proposed control system is depicted in Figure 5. 


\section{Stability Analysis}

For any given $p>0$, the closed sets can be defined as follows:

$$
\begin{gathered}
\Omega_{1}=\left\{\left(S_{1}, q_{11}, \ldots, q_{31}\right): V_{1} \leq p\right\}, \\
\Omega_{2}=\left\{\left(S_{1}, S_{2}, \widehat{\theta}_{1}, y_{2}, \widehat{g}_{1}, q_{11}, \ldots, q_{31}, q_{22}, q_{32}\right): V_{2} \leq p\right\}, \\
\Omega_{3}=\left\{\left(s_{1}, \ldots, S_{3}, \widehat{\theta}_{1}, \widehat{\theta}_{2}, y_{2}, y_{3}, \widehat{g}_{1}, \widehat{g}_{2}, q_{11}, \ldots,\right.\right. \\
\left.\left.q_{31}, q_{22}, q_{32}, q_{33}\right): V_{3} \leq p\right\}, \\
\Omega_{4}=\left\{\left(S_{1}, \ldots, S_{4}, \widehat{\theta}_{1}, \ldots, \widehat{\theta}_{3}, y_{2}, y_{3}, \widehat{g}_{1}, \ldots, \widehat{g}_{3},\right.\right. \\
\left.\left.q_{11}, \ldots, q_{31}, q_{22}, q_{32}, q_{33}, q_{44}\right): V_{4} \leq p\right\} .
\end{gathered}
$$

Theorem 2. Suppose that the control law in (47) and (55) with adaptive law in (32), (33), (48), (49), (56), and (57) is applied to the PMSM (4), Assumption 1 stands, and if there exists a positive constant $p$ that the initial condition satisfies $V_{4} \leq p$ and the design constants $k_{i}, \tau_{i}$, and $a_{0}$ satisfy the following conditions:

$$
\begin{gathered}
k_{1} \geq 1, \quad k_{2} \geq 2 g_{M 1}+1+a_{0}, \\
k_{3} \geq \frac{1}{4} g_{M 2}+1+a_{0}, \quad k_{4} \geq \frac{1}{4} g_{M 3}+1+a_{0}, \\
\frac{1}{\tau_{i}} \geq \frac{1}{4} g_{M i}+1+a_{0}, \quad a_{0} \leq \frac{1}{2} \min _{1 \leq i \leq 3}\left\{\gamma_{i}, \Gamma_{i}, m_{i}, c_{i}\right\},
\end{gathered}
$$

then the closed-loop control system is semiglobal uniformly ultimately bounded, and the output tracking error converges to a neighborhood of zero.

Proof. Define the following Lyapunov function candidate for the fourth step:

$$
\begin{aligned}
V= & \frac{1}{2}\left(\sum_{j=1}^{4} S_{j}^{2}+\sum_{j=2}^{3} y_{j}^{2}+\sum_{j=1}^{3} \frac{1}{\gamma_{j}} \widetilde{\theta}_{j}^{T} \widetilde{\theta}_{j}+\sum_{j=1}^{3} \frac{1}{\Gamma_{j}} \widetilde{g}_{j}^{2}\right) \\
& +\sum_{j=1}^{3} \int_{t-\tau_{j}}^{t} S_{1}^{2}(\tau) q_{j 1}^{2}\left(\bar{S}_{1}(\tau)\right) d \tau \\
& +\sum_{j=2}^{3} \int_{t-\tau_{j}}^{t} S_{2}^{2}(\tau) q_{j 2}^{2}\left(\bar{S}_{2}(\tau)\right) d \tau \\
& +\int_{t-\tau_{3}}^{t} S_{3}^{2}(\tau) q_{33}^{2}\left(\bar{S}_{3}(\tau)\right) d \tau \\
& +\int_{t-\tau_{4}}^{t} S_{4}^{2}(\tau) q_{44}^{2}\left(S_{4}(\tau)\right) d \tau .
\end{aligned}
$$

For the term $-m_{j} \widetilde{\theta}_{j}^{T} \widehat{\theta}_{j}$, one has $-m_{j} \widetilde{\theta}_{j}^{T} \widehat{\theta}_{j}=-m_{j} \widetilde{\theta}_{j}^{T}\left(\widetilde{\theta}_{j}+\right.$ $\left.\theta_{j}\right) \leq-(1 / 2) m_{j}\left\|\widetilde{\theta}_{j}\right\|^{2}+(1 / 2) m_{j}\left\|\theta_{j}\right\|^{2}$. Similarly, $-c_{j} \widetilde{g}_{j} \widehat{g}_{j} \leq$
$-(1 / 2) c_{j} \tilde{g}_{j}^{2}+(1 / 2) c_{j} g_{j}^{2}$ holds. Consequently, the derivative of $V$ is

$$
\begin{aligned}
\dot{V}= & -\sum_{j=1}^{4} k_{j} S_{j}^{2}+\sum_{j=1}^{2} S_{j}^{2}-\sum_{j=1}^{3} \frac{1}{2} m_{j}\left\|\widetilde{\theta}_{j}\right\|^{2}-\sum_{j=1}^{3} \frac{1}{2} c_{j} \widetilde{g}_{j}^{2} \\
& +\sum_{j=2}^{3}\left(1-\frac{1}{\tau_{j}}\right) y_{j}^{2}+\frac{1}{2} y_{2}^{2}+\frac{1}{2} g_{1} y_{3}^{2}-\frac{1}{2} S_{2}^{2} \widetilde{g}_{1} \\
& +\sum_{j=1}^{4} D_{j}+\sum_{j=1}^{3} \frac{1}{4} \vartheta_{j}^{2}+\sum_{j=2}^{3} \frac{1}{4} B_{j}^{2}+\sum_{j=1}^{3} \frac{1}{2} m_{j}\left\|\theta_{j}\right\|^{2} \\
& +\sum_{j=1}^{3} \frac{1}{2} c_{j} g_{j}^{2} \leq-a_{0} V+b_{0},
\end{aligned}
$$

where $b_{0}=\sum_{j=1}^{3}(1 / 4) \vartheta_{j}^{2}+\sum_{j=2}^{3}(1 / 4) B_{j}^{2}+\sum_{j=1}^{3}(1 / 2) m_{j}\left\|\theta_{j}\right\|^{2}+$ $\sum_{j=1}^{3}(1 / 2) c_{j} g_{j}^{2}$.

Furthermore, (62) implies that

$$
0 \leq V(t) \leq \frac{b_{0}}{a_{0}}+\left(V(0)-\frac{b_{0}}{a_{0}}\right) e^{-a_{0} t} \leq \frac{b_{0}}{a_{0}}+V(0)
$$

By Lyapunov function, it is proved that all the signals $S_{j}, y_{j}, \widetilde{\theta}_{j}$, and $\widetilde{g}_{j}$ in the closed-loop system are uniformly ultimately bounded. Consequently, $x_{i}, \alpha_{i}$, and $\alpha_{i f}$ are also uniformly ultimately bounded.

\section{Simulation}

To illustrate the effectiveness of the proposed controller, the simulation will be done for the PMSM with the parameters: $J=0.00379 \mathrm{Kgm}^{2}, R_{S}=0.68 \Omega, L_{d}=0.00315 \mathrm{H}, L_{q}=$ $0.00285 \mathrm{H}, B=0.001158 \mathrm{Nm} /(\mathrm{rad} / \mathrm{s}), \Phi=0.1245 \mathrm{~Wb}, n_{p}=3$, $T_{L}=1.5, q_{11}=1, q_{21}=1-\sqrt{2-S_{1}^{2}}, q_{31}=\left|S_{1}\right|, q_{22}=\left|S_{2}\right|, q_{32}=$ $\sqrt{2-S_{2}^{2}}, q_{33}=1, q_{44}=1, d_{1}=d_{2}=d_{3}=d_{4}=0.2 \times x_{1}^{3} \times \sin ^{5}(t)$, $\Delta f_{1}\left(x_{1}\left(t-\tau_{1}\right)\right)=0.05 \sin \left(x_{1}\left(t-\tau_{1}\right)\right), \Delta f_{2}\left(\bar{x}_{2}\left(t-\tau_{2}\right)\right)=x_{1}(t-$ $\left.\tau_{2}\right) x_{2}\left(t-\tau_{2}\right), \Delta f_{3}\left(\bar{x}_{3}\left(t-\tau_{3}\right)\right)=x_{1}\left(t-\tau_{3}\right) x_{2}\left(t-\tau_{3}\right) x_{3}\left(t-\tau_{3}\right)$, $\Delta f_{4}\left(x_{4}\left(t-\tau_{4}\right)\right)=0.05 \sin \left(x_{4}\left(t-\tau_{4}\right)\right), \tau_{1}=\tau_{2}=\tau_{3}=\tau_{4}=0.4 \mathrm{~s}$.

The simulation is running under the assumption that the system parameters and nonlinear functions are unknown. The initial conditions of system $x_{1}(0)=x_{2}(0)=x_{3}(0)=$ $x_{4}(0)=0$. Then, the reference signal is taken as $y_{r}=\sin 3 t$, the control parameters are chosen as follows: $k_{1}=k_{2}=25$, $k_{3}=k_{4}=20, \Gamma_{1}=\Gamma_{2}=\Gamma_{3}=0.3, c_{1}=c_{2}=c_{3}=0.9$, $\tau_{2}=0.01, \tau_{3}=0.02, \gamma_{1}=\gamma_{2}=\gamma_{3}=25, m_{1}=m_{2}=m_{3}=0.1$, $\eta_{1}=\eta_{2}=\eta_{3}=0.001$, and RBFNN is chosen in the following way. Neural network $\theta_{j}^{T} \xi_{j}, j=1,2,3$, contains nine nodes with centers spaced evenly in the interval $[-5,5]$ and width being equal to two.

With the proposed RBFNN-based adaptive dynamic surface control, from Figures 6 and 7, it is observed that the tracking performance is satisfactory and the tracking error quickly converges to zero in a short time for PMSM system in the presence of unknown parameters, disturbances, and uncertain time delays. The final controllers $u_{q}$ and $q_{d}$ are 


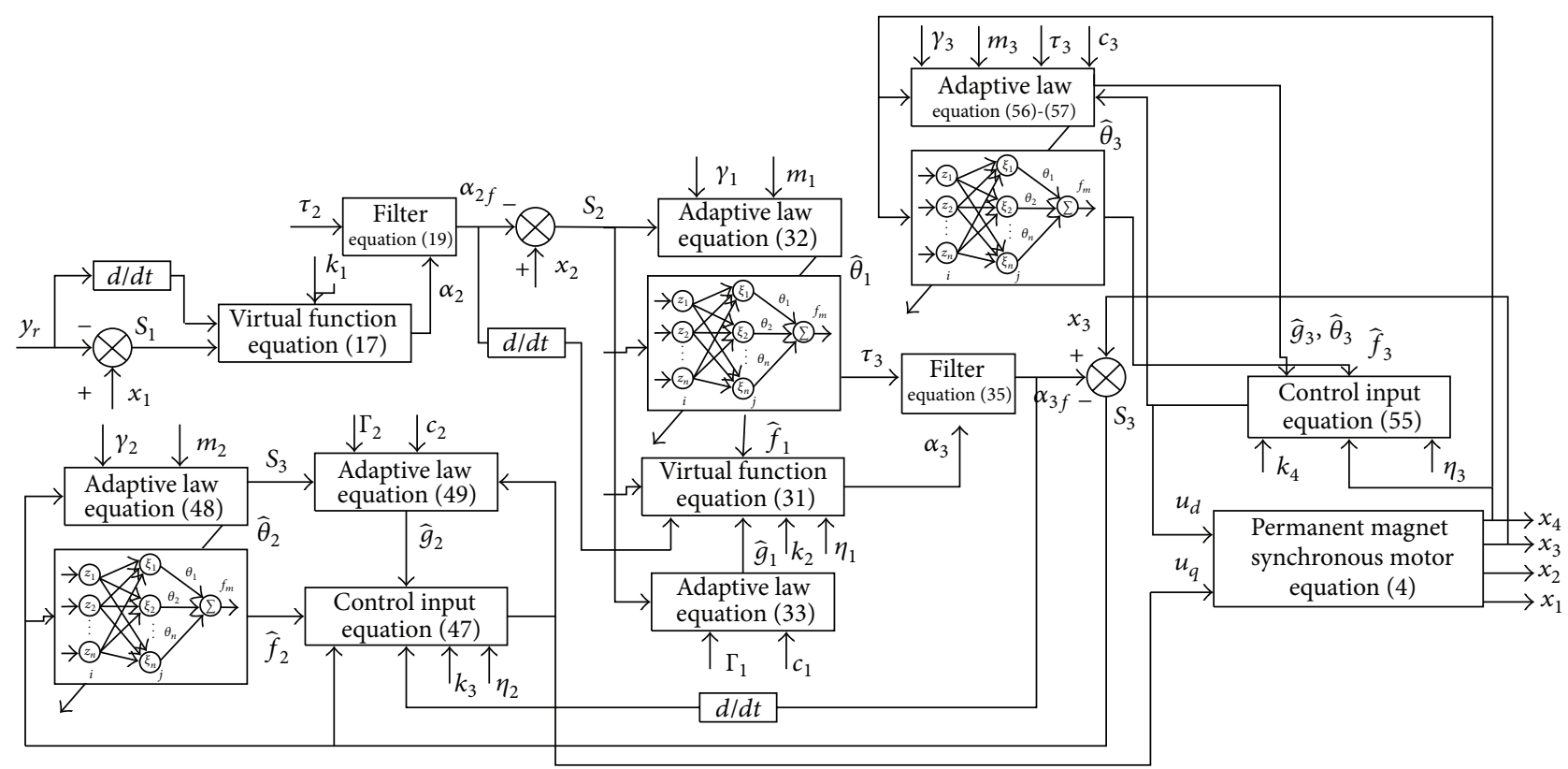

FIGURE 5: Block diagram of the adaptive DSC system based on RBFNN for PMSM.
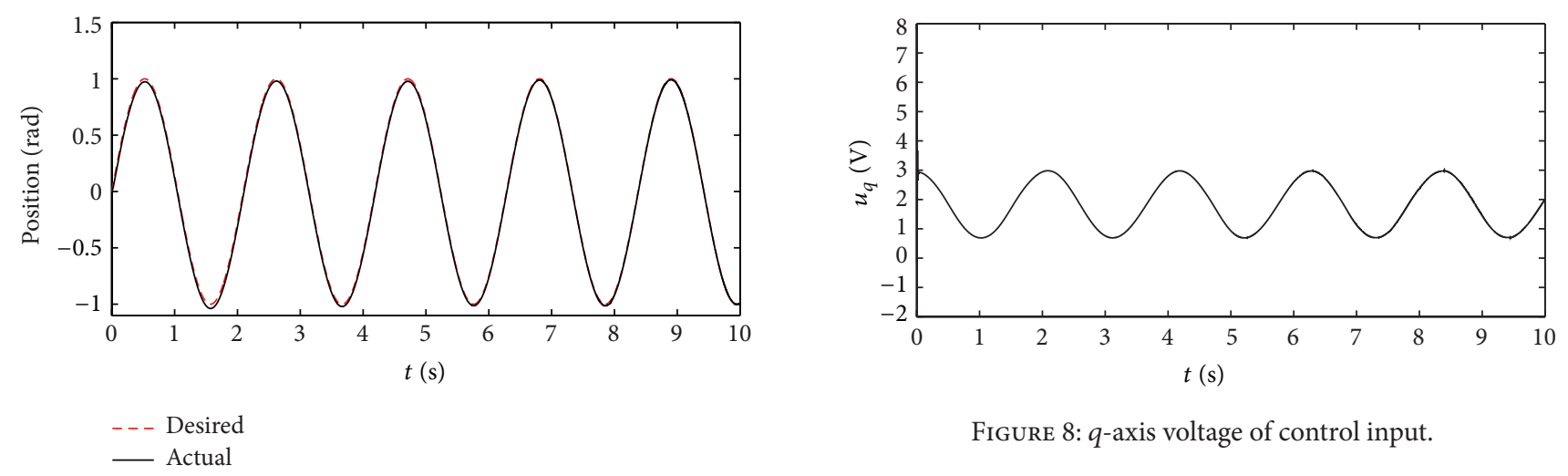

FIGURE 8: $q$-axis voltage of control input.

FIgURE 6: Output $x_{1}$ (solid line) follows desired trajectory $y_{r}$ (dashed line).
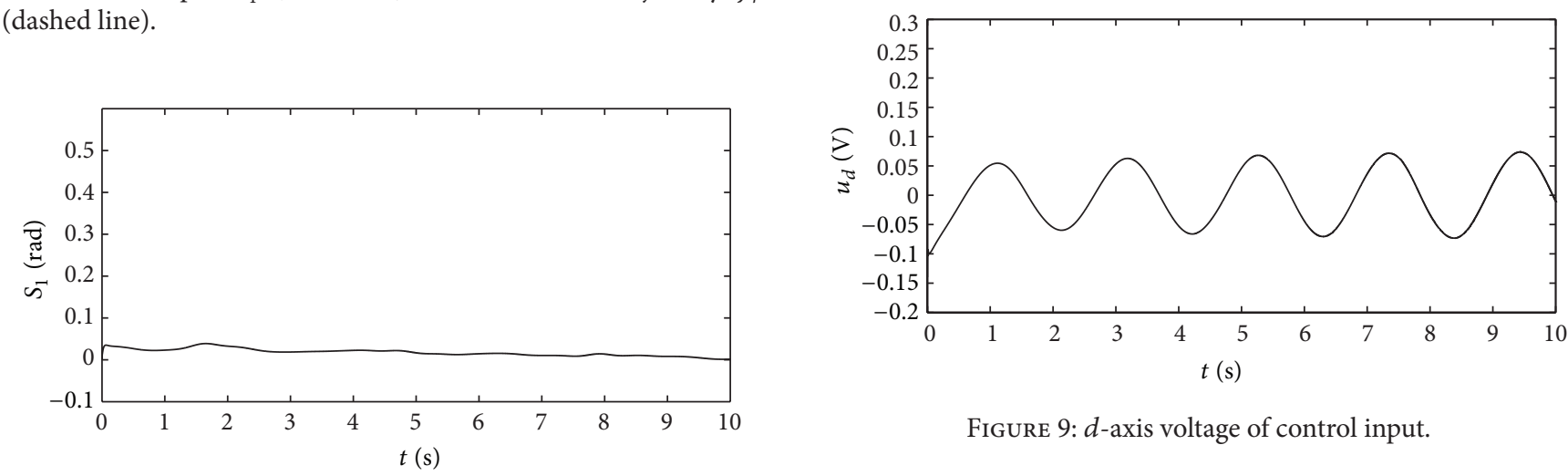

FIGURE 9: $d$-axis voltage of control input.

Figure 7: The rotor position tracking error.

shown in Figures 8 and 9. Moreover, the robustness of the proposed controllers against uncertainty in system parameters and disturbance is analyzed by simulation. According to

Figure 10, it is seen clearly that all state variables are bounded and convergent. Meanwhile, by contrast with Figure 3, the proposed controller can suppress the chaos of PMSM. Therefore, these simulation results demonstrate the effectiveness of the proposed control in achieving its objective. 

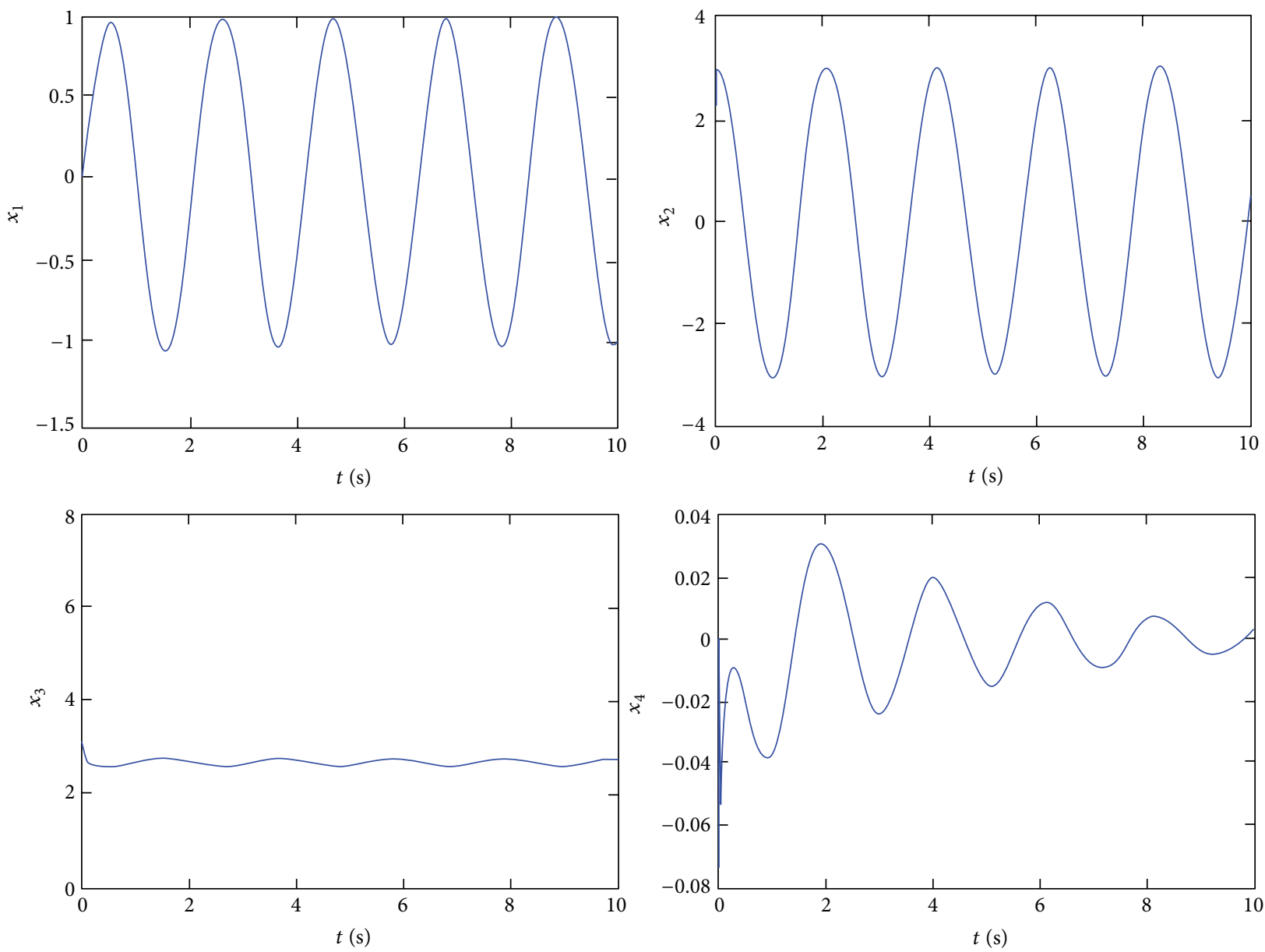

FIGURE 10: The curves of system state variables when utilizing the proposed controller.

\section{Conclusion}

A novel adaptive DSC based on the RBFNN is presented for the fourth-order chaotic PMSM system with uncertain time delays so that the first state can track a given trajectory with little time. The unknown PMSM parameters, disturbances, and uncertain time delays are all considered. Compared with the traditional backstepping approach, the main advantage of the proposed method is its simplicity without repetitive differentiation of the nonlinear functions under the condition. It is noted that the system has excellent tracking performance and antidisturbing by choosing appropriate design parameters. Finally, the simulation results are provided to demonstrate the effectiveness and robustness against the unknown parameters, disturbances, chaos, and uncertain time delays.

\section{Conflict of Interests}

The authors declare that there is no conflict of interests regarding the publication of this paper.

\section{Acknowledgments}

This work is supported by the Natural Science Foundation of China (Grants nos. 51375506, 50905189), the Specialized
Research Fund for the Doctoral Program of Higher Education (Grant no. 20100191110008), China Postdoctoral Science Foundation funded project (Grant no. 2013M542258), and the Par-Eu Scholars Program Special Foundation of Chongqing. The authors also gratefully acknowledge the helpful comments and suggestions of the reviewers, which have improved the presentation.

\section{References}

[1] C. Elmas and O. Ustun, "A hybrid controller for the speed control of a permanent magnet synchronous motor drive," Control Engineering Practice, vol. 16, no. 3, pp. 260-270, 2008.

[2] K. Hartani, Y. Miloud, and A. Miloudi, "Improved direct torque control of permanent magnet synchronous electrical vehicle motor with proportional-integral resistance estimator," Journal of Electrical Engineering \& Technology, vol. 5, no. 3, pp. 451-461, 2010.

[3] J. Yu, J. Gao, Y. Ma, H. Yu, and S. Pan, "Robust adaptive fuzzy control of chaos in the permanent magnet synchronous motor," Discrete Dynamics in Nature and Society, vol. 2010, Article ID 269283, 13 pages, 2010.

[4] M. Zribi, A. Oteafy, and N. Smaoui, "Controlling chaos in the permanent magnet synchronous motor," Chaos, Solitons \& Fractals, vol. 41, no. 3, pp. 1266-1276, 2009. 
[5] N. Mitrakis, J. Theocharis, and V. Petridis, "A multilayered neuro-fuzzy classifier with self-organizing properties," Fuzzy Sets and Systems, vol. 159, no. 23, pp. 3132-3159, 2008.

[6] C. Zhang, H. Liu, S. Chen, and F. Wang, "Application of neural networks for permanent magnet synchronous motor direct torque control," Journal of Systems Engineering and Electronics, vol. 19 , no. 3, pp. 555-561, 2008.

[7] C. Elmas, O. Ustun, and H. H. Sayan, "A neuro-fuzzy controller for speed control of a permanent magnet synchronous motor drive," Expert Systems with Applications, vol. 34, no. 1, pp. 657664, 2008.

[8] A. Mezouar, M. K. Fellah, and S. Hadjeri, "Adaptive sliding mode observer for induction motor using two-time-scale approach," Electric Power Systems Research, vol. 77, no. 5-6, pp. 604-618, 2007.

[9] Y. Feng, J. Zheng, X. Yu, and N. V. Truong, "Hybrid terminal sliding-mode observer design method for a permanent-magnet synchronous motor control system," IEEE Transactions on Industrial Electronics, vol. 56, no. 9, pp. 3424-3431, 2009.

[10] J. Yu, B. Chen, and H. Yu, "Position tracking control of induction motors via adaptive fuzzy backstepping," Energy Conversion and Management, vol. 51, no. 11, pp. 2345-2352, 2010.

[11] P. Yip and J. Hedrick, "Adaptive dynamic surface control: a simplified algorithm for adaptive backstepping control of nonlinear systems," International Journal of Control, vol. 71, no. 5, pp. 959979, 1998.

[12] Z. Wang, "Synchronization of an uncertain fractional-order chaotic system via backstepping sliding mode control," Discrete Dynamics in Nature and Society, vol. 2013, Article ID 732503, 6 pages, 2013.

[13] D. Swaroop, J. K. Hedrick, P. P. Yip, and J. C. Gerdes, "Dynamic surface control for a class of nonlinear systems," IEEE Transactions on Automatic Control, vol. 45, no. 10, pp. 1893-1899, 2000.

[14] D. Wang and J. Huang, "Neural network-based adaptive dynamic surface control for a class of uncertain nonlinear systems in strict-feedback form," IEEE Transactions on Neural Networks, vol. 16, no. 1, pp. 195-202, 2005.

[15] T. Zhang and S. Ge, "Adaptive dynamic surface control of nonlinear systems with unknown dead zone in pure feedback form," Automatica, vol. 44, no. 7, pp. 1895-1903, 2008.

[16] D. Wei, X. S. Luo, B. Wang, and J. Fang, "Robust adaptive dynamic surface control of chaos in permanent magnet synchronous motor," Physics Letters A, vol. 363, no. 1-2, pp. 71-77, 2007.

[17] S. J. Yoo, J. B. Park, and Y. H. Choi, "Adaptive neural control for a class of strict-feedback nonlinear systems with state time delays," IEEE Transactions on Neural Networks, vol. 20, no. 7, pp. 1209-1215, 2009.

[18] S. J. Yoo and J. B. Park, "Neural-network-based decentralized adaptive control for a class of large-scale nonlinear systems with unknown time-varying delays," IEEE Transactions on Systems, Man, and Cybernetics B, vol. 39, no. 5, pp. 1316-1323, 2009.

[19] S. Han and J. Lee, "Precise positioning of nonsmooth dynamic systems using fuzzy wavelet echo state networks and dynamic surface sliding mode control," IEEE Transactions on Industrial Electronics, vol. 60, no. 11, pp. 5124-5136, 2013.

[20] J. Na, X. Ren, G. Herrmann, and Z. Qiao, "Adaptive neural dynamic surface control for servo systems with unknown deadzone," Control Engineering Practice, vol. 19, no. 11, pp. 1328-1343, 2011.

[21] W. Leonhard, Control of Electrical Drives, Springer, Berlin, Germany, 1985.
[22] J. Yu, J. Gao, Y. Ma, and H. Yu, "Adaptive fuzzy tracking control for a permanent magnet synchronous motor via backstepping approach," Mathematical Problems in Engineering, vol. 2010, Article ID 391846, 13 pages, 2010. 


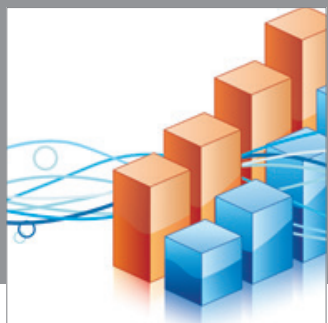

Advances in

Operations Research

mansans

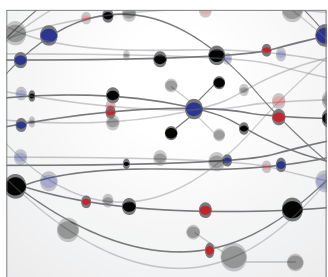

The Scientific World Journal
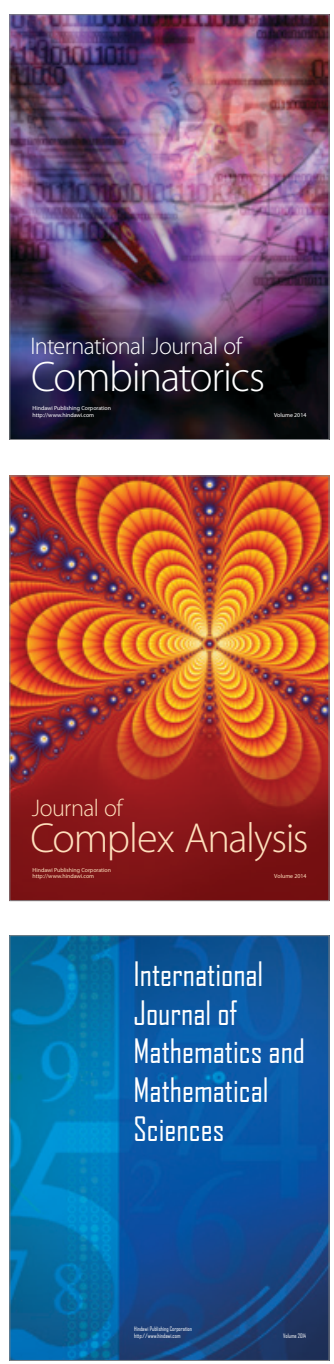
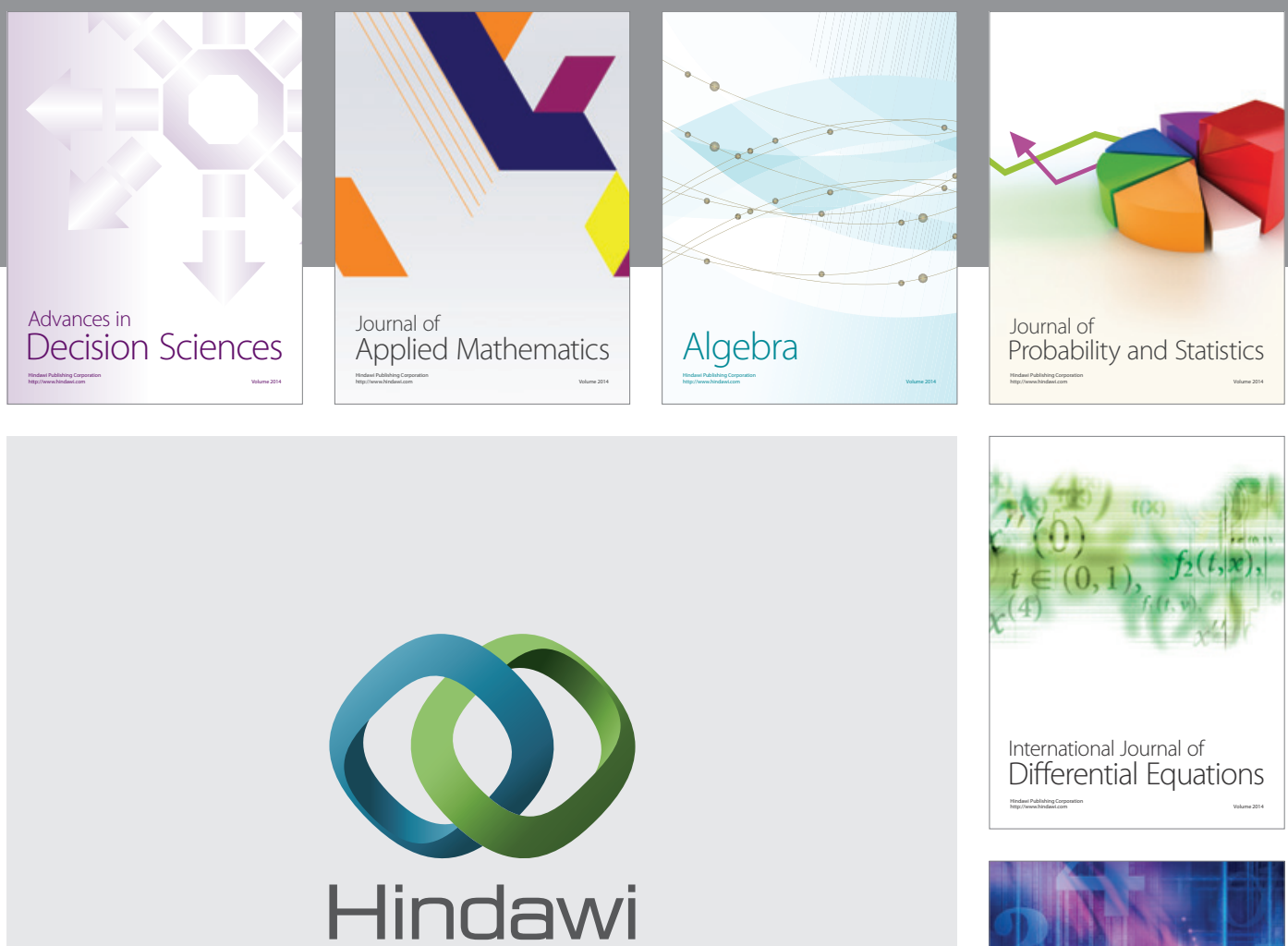

Submit your manuscripts at http://www.hindawi.com
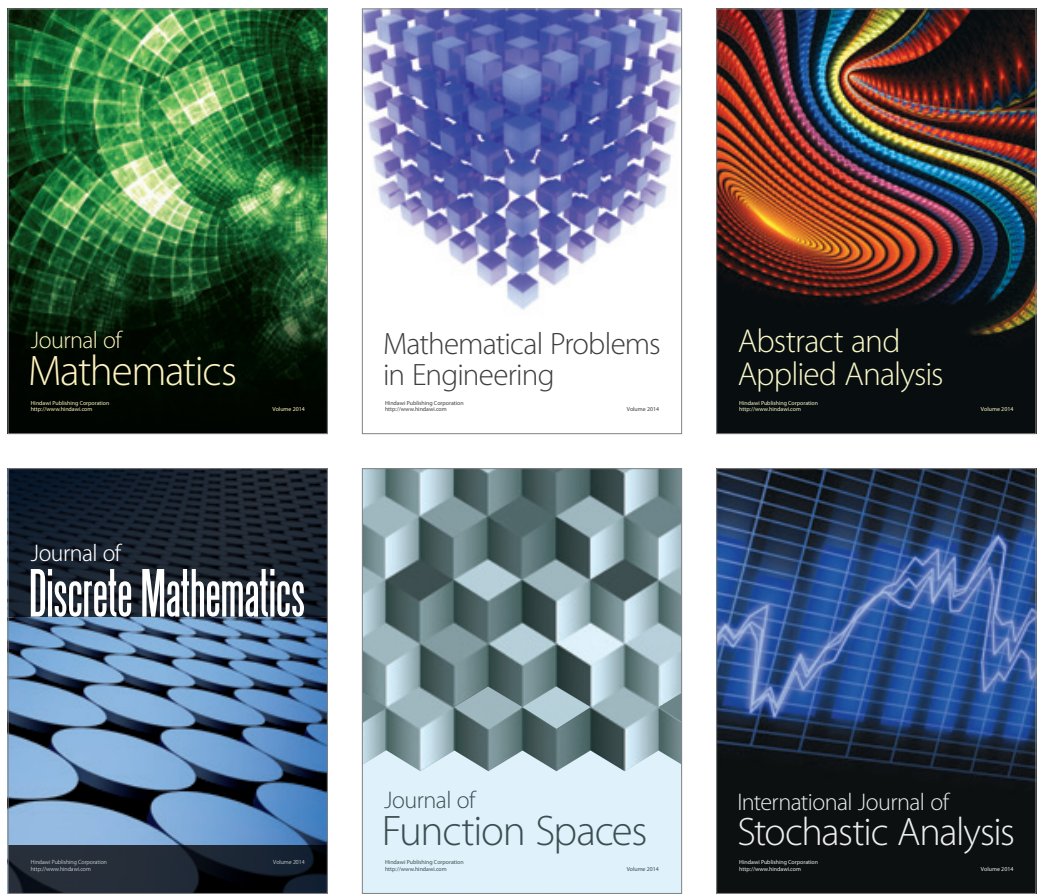

Journal of

Function Spaces

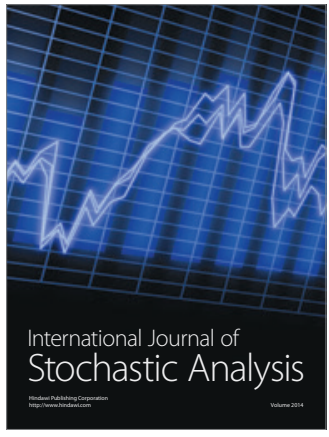

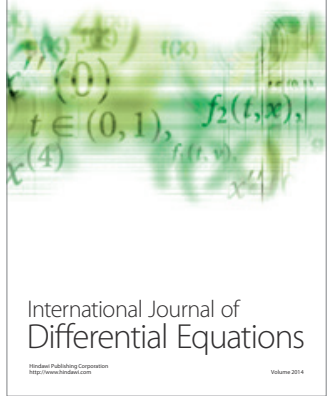
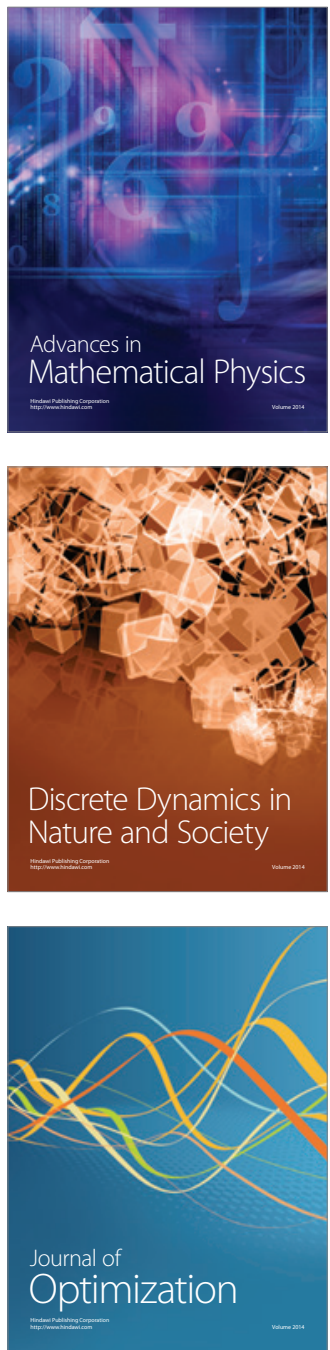